\title{
INFECÇÕES HOSPITALARES BACTERIANAS EM UNIDADE DE TRANSPLANTE HEPÁTICO
}

\author{
Bacterial nosocomial infections in a liver transplantation unit
}

\begin{abstract}
Júlio Cezar Uili Coelho,TCBC-PR', Mônica Beatriz Parolin², Jorge Eduardo Fouto Matias ${ }^{3}$, Alexandre Coutinho Teixeira de Freitas, TCBC-PR ${ }^{3}$, André Ricardo Dall'Oglio Tolazzi ${ }^{4}$, Marta Francisca de Fátima Fragoso ${ }^{5}$, Maria Edutânia Skroski Castro.
\end{abstract}

\section{RESUMO}

Objetivo: A incidência de infecção após transplante hepático permanece elevada, sendo maior se comparada aos transplantes de outros órgãos sólidos. Este estudo tem por objetivo apresentar a incidência, distribuição topográfica e os microorganismos mais freqüentemente encontrados nas infecções hospitalares em uma unidade de transplante hepático de um Hospital Universitário. Métodos: os prontuários de 1963 pacientes admitidos para transplante hepático ou por complicações pré ou pós-transplante foram avaliados prospectivamente, utilizando os critérios diagnósticos de infecções nosocomiais instituídos pelo Centro de Controle e Prevenção de Doenças dos Estados Unidos. Durante o período de estudo foram realizados 175 transplantes hepáticos, sendo 148 com enxerto cadavérico e 27 transplantes intervivos. Resultados: infecções hospitalares foram diagnosticadas em 79 pacientes, representando 5,5\% do total das internações. A infecção de sítio cirúrgico apresentou-se com maior incidência ( $27,7 \%$ ou 30 casos), seguida pela pneumonia (20,3\%), infecções do aparelho digestivo $(15,7 \%)$ e relacionadas à corrente sangüínea (12\%). Dos microorganismos isolados nas 79 culturas positivas, os mais freqüentes foram: Staphylococcus sp (40,5\%), Enterococcus faecalis (13,9\%), Klebsiella pneumoneae e Escherichia coli (7,6\% cada). Das infecções causadas por bactérias multirresistentes (43\% do total), Staphylococcus aureus resistente a meticilina (MRSA) foi o mais prevalente (22 infecções). A mortalidade global relacionada às infecções hospitalares foi de 22,7\% (18 pacientes) e a infecção de maior letalidade esteve relacionada à corrente sangüínea (30,7\%). Conclusão: o local mais comum de infecção bacteriana em pacientes de serviço de transplante hepático é o sítio cirúrgico e o local de maior letalidade é a infecção da corrente sangüínea. As bactérias isoladas mais freqüentes são Staphylococcus sp e Enterococcus faecalis, sendo que um número expressivo destas bactérias são multirresistentes.

Descritores: Infecção hospitalar, Bactéria, Pneumonia, Transplante hepático, Hepatopatia.

\footnotetext{
Trabalho realizado na Unidade de Transplante Hepático e Serviço de Controle de Infecção Hospitalar do Hospital de Clínicas da Universidade Federal do Paraná.

1 Professor Titular e Chefe do Serviço de Cirurgia do Aparelho Digestivo e Unidade de Transplante Hepático do Hospital de Clínicas (HC) da Universidade Federal do Paraná (UFPR);

${ }^{2}$ Médica da Unidade de Transplante Hepático - HC - UFPR;

${ }^{3}$ Médico do Serviço de Cirurgia do Aparelho Digestivo e Unidade de Transplante Hepático HC - UFPR;

${ }^{4}$ Médico Residente do Serviço de Cirurgia do Aparelho Digestivo - HC - UFPR;

${ }^{5}$ Médica do Serviço de Controle de Infecção Hospitalar - HC - UFPR;

${ }^{6}$ Enfermeira do Serviço de Controle de Infecção Hospitalar - HC - UFPR;

Endereço para correspondência: Dr. Júlio Coelho. Rua Bento Viana, 1140 - Ap.2002 CEP 80240-110 - Curitiba-PR - Fone/Fax: (41) 322-3789 - E-mail: juliocoelho@bbs2.sul.com.br Recebido em: 30/06/2004 Aceito:06/09/2004
}

\section{INTRODUÇÃO}

Desde o primeiro transplante hepático $(\mathrm{TH})$ ortotópico realizado por Thomas Starzl em 1963, as infecções, nas suas mais variadas formas e etiologias, permanecem como uma constante e importante ameaça aos doentes candidatos ou já submetidos a esse tipo de tratamento. ${ }^{1-5}$ Embora a taxa de mortalidade relacionada às infecções dos pacientes pós-transplante hepático tenha diminuído substancialmente, a incidência de infecção após esse procedimento permanece elevada, sendo maior se comparada aos transplantes de outros órgãos sólidos. Dois terços dos pacientes submetidos ao TH apresentam pelo menos um episódio de infecção na sua evolução, sendo as infecções bacterianas as causas mais comuns. ${ }^{6}$

A incidência de infecção e os seus agentes etiológicos variam conforme o tipo de operação e as condições econômicas do país. Existem poucas avaliações sobre as infecções em serviços de transplante hepático no Brasil. O presente estudo tem por objetivo apresentar a incidência, distribuição topográfica e os microorganismos mais freqüentemente encontrados nas infecções hospitalares (IH) em pacientes internados em uma unidade de transplante hepático de um Hospital Universitário. 


\section{MÉTODOS}

Os pacientes internados no Serviço de Transplante Hepático do Hospital de Clínicas da Universidade Federal do Paraná foram prospectivamente avaliados no período de 1 de janeiro de 1998 a 31 de dezembro de 2002. Foram estudadas 1.963 admissões hospitalares de pacientes submetidos a transplante hepático, ou que apresentaram complicações pré ou pós-transplante. Nesse período, houve um total de 175 transplantes hepáticos (106 receptores masculinos e 69 femininos), sendo 148 com enxerto cadavérico e 27 transplantes intervivos (15 receptores masculinos e 12 femininos).

As informações coletadas a respeito das infecções hospitalares basearam-se em dados microbiológicos, utilização de antimicrobianos, informações prestadas pela equipe médica responsável, consulta ao paciente e inspeção do local por profissionais do Serviço de Controle de Infecção Hospitalar, bem como coleta de dados dos prontuários médicos. Os critérios diagnósticos adotados para as infecções nosocomiais foram os instituídos pelo Centro de Controle e Prevenção de Doenças dos Estados Unidos. ${ }^{1,3,5}$ As infecções de ferida cirúrgica (SSI) foram classificadas em infecções de sítio cirúrgico superficial, profundo, de órgão ou cavidade, diagnosticadas nos primeiros 30 dias pós-operatórios. Infecções do trato respiratório inferior (LRI) incluíram as traqueobronquites, empiemas pleurais, abscessos pulmonares ou outras, porém sem evidência de pneumonia. As pneumonias representaram um grupo à parte. Infecções primárias da corrente sangüínea (BSI) foram aquelas confirmadas laboratorialmente (isolamento do patógeno na hemocultura, não relacionado com infecção em outro sítio) ou sepse clínica em que não se identificou o agente etiológico e sem outra causa conhecida. As infecções do trato urinário (UTI) englobaram as infecções urinárias sintomáticas, bacteriúria assintomática, entre outras formas. Infecções do sistema cardiovascular (CVS) envolveram infecções relacionadas a cateteres vasculares, do sistema arterial ou venoso (flebites), endocardite, miocardite, pericardite ou mediastinite. As infecções do aparelho digestivo (AD) representaram aquelas do trato gastrointestinal propriamente dito ou outras da cavidade abdominal (órgão ou espaço) como hepatite, colangite e abscesso intra-abdominal. No período pósoperatório de cirurgia abdominal, como no $\mathrm{TH}$, as infecções do $\mathrm{AD}$, muitas vezes, ocorriam simultaneamente às de SSI e eram classificadas em ambas as categorias. Outras categorias de infecção presentes nesse estudo foram infecções oftalmológicas e otorrinolaringológicas (EENT) e de pele ou partes moles (SST) não enquadradas nas categorias anteriormente citadas.

Os pacientes foram avaliados quanto à distribuição sazonal, sítio das infecções, identificação dos microorganismos, taxa de letalidade e mortalidade das IH.

\section{RESULTADOS}

No período estudado, setenta e nove pacientes apresentaram IH, representando 109 (5,5\%) do total de 1963 internações na unidade de transplante hepático. A distribuição mensal das IH foi bastante irregular, sendo que os meses de maior incidência foram junho $(12,5 \%)$, agosto $(12,3 \%)$ e novembro $(10,3 \%)$; e os de menor incidência foram janeiro $(3,2 \%)$, março $(1,4 \%)$ e julho $(2,9 \%)$ (Gráfico 1).

№ DE CASOS

$(\mathrm{N}=109)$

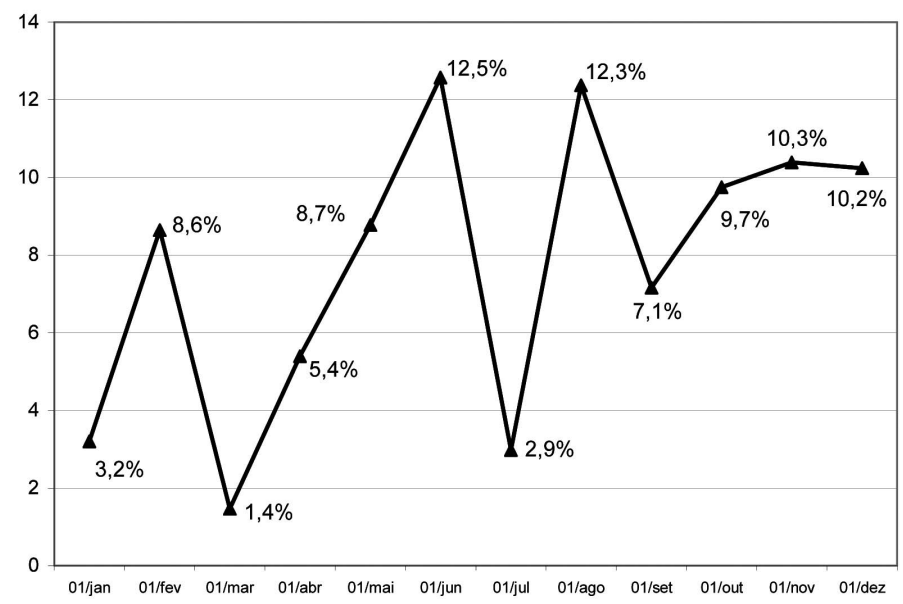

Gráfico 1: Incidência mensal das infecções hospitalare

Em relação ao local de IH, as SSI foram as mais freqüentes $(27,7 \%$ ou 30 casos), seguida pelas pneumonias em $20,3 \%$ ( 22 casos), AD em $15,7 \%$ (17 casos), BSI em 12\% (13 casos), UTI em 11,1\% (12 casos), CVS em 6,5\% (7 casos), LRI e EENT em 2,8\% (3 casos) cada, e SST em $0,9 \%$ (1 caso) (Gráfico 2).

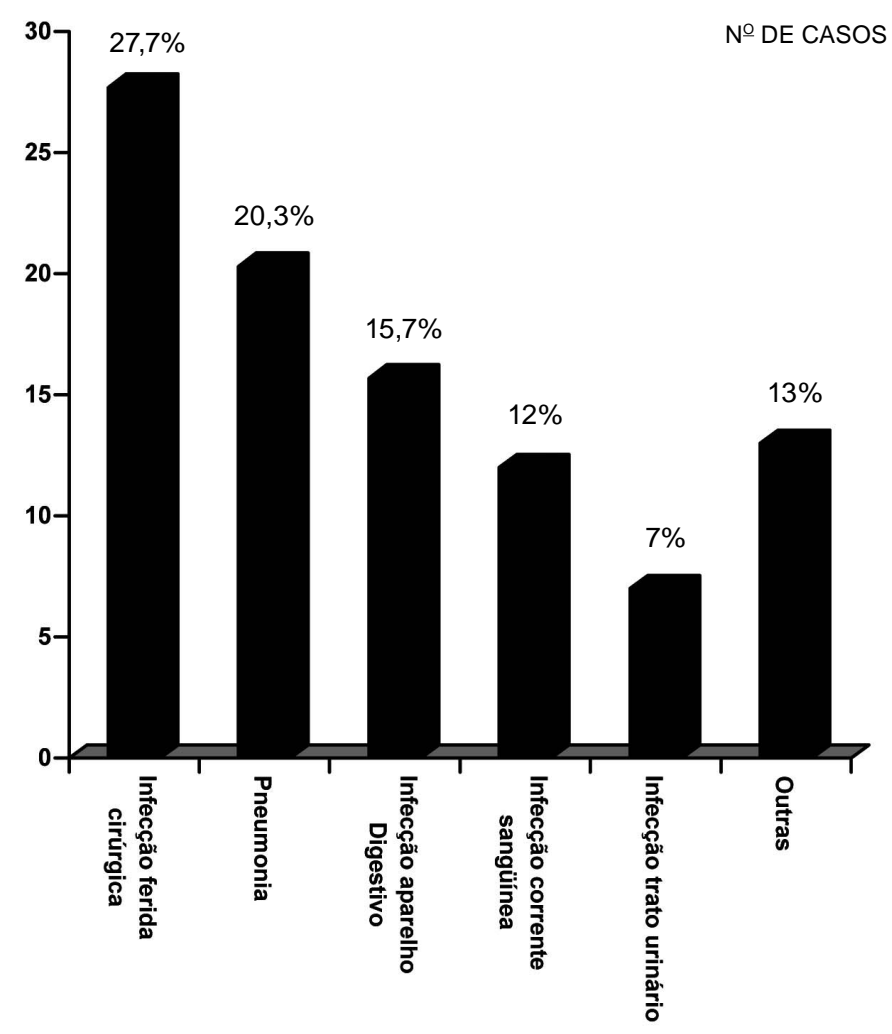

Gráfico 2: Sítios de infecção hospitalar em percentagem 
Dos microorganismos isolados nas 79 culturas positivas, os mais freqüentes foram: Staphylococcus $s p$ em 32 casos (40,5\%), Enterococcus faecalis em $11(13,9 \%)$, Klebsiella pneumoneae em 6 (7,6\%), Escherichia coli em 6 (7,6\%), Acinetobacter $s p$ em 5 (6,3\%), Pseudomonas aeruginosa em 5 (6,3\%) e Candida sp em $3(3,8 \%)$. Das infecções causadas por bactérias multirresistentes (MR) e pan-resistentes (PR), Staphylococcus aureus resistente à meticilina (MRSA) foi isolado em 22 infecções $(27,8 \%)$, Staphylococcus coagulase-negativo resistente a meticilina (MRSE-ENPC) em 6 (7,5\%), Klebsiella pneumoneae-MR em 3 (3,8\%), Pseudomonas-MR em 2 (2,5\%) e Acinetobacter baumaniiPR em 1 caso $(1,2 \%)$ (Tabela 1$)$.

Tabela 1: Freqüência dos microorganismos isolados por cultura

\begin{tabular}{|c|c|}
\hline Microorganismos & $\begin{array}{l}\text { Número } \\
\text { de casos }\end{array}$ \\
\hline Staphylococcus aureus (MSSA)* & 5 \\
\hline Staphylococcus aureus (MRSA)* & 22 \\
\hline Staphylococcus epidermidis e não produtor de coagulase (MRSE-ENPC) $)^{*}$ & 6 \\
\hline Enterococcus faecalis & 11 \\
\hline Enterococcus sp & 2 \\
\hline Klebsiella pneumoniae & 3 \\
\hline Klebsiella pneumoniae-MR (ESBL)* & 3 \\
\hline Klebsiella oxytoca & 1 \\
\hline Klebsiella ozanae & 1 \\
\hline Escherichia coli & 6 \\
\hline Acinetobacter baumanii & 3 \\
\hline Acinetobacter baumanii-PR* & 1 \\
\hline Acinetobacter Iwoffi & 1 \\
\hline Pseudomonas aeruginosa & 3 \\
\hline Pseudomonas aeruginosa-MR* & 2 \\
\hline Cândida sp & 3 \\
\hline Enterobacter cloacae & 2 \\
\hline Enterobacter aerogenes & 1 \\
\hline Corynebacterium sp & 1 \\
\hline Citrobacter freudii & 1 \\
\hline Stenotrophomonas maltophilia & 1 \\
\hline Total & 79 \\
\hline
\end{tabular}

* MSSA: Staphylococcus aureus sensível à meticilina; MRSA Staphylococcus aureus resistentes à meticilina; MRSE-ENPC Estafilococo não produtor de coagulase e resistente à meticilina; MR: forma multirresistente (resistente à cefalosporinas e sensível à carbapenêmicos); PR: forma pan-resistente (resistente a carbapenêmicos e sensível a polimixina $B$ ).

Em relação aos microorganismos mais freqüentemente isolados nas infecções de ferida cirúrgica (SSI) - 24 culturas positivas - Staphylococcus sp foi encontrado em mais da metade dos casos (54,1\% ou 13 casos), sendo o Staphylococcus aureus resistente à meticilina (MRSA) isolado em 9 casos, Staphylococcus aureus sensível à meticilina (MSSA) em 2 e Staphylococcus epidermidis resistente à meticilina (MRSE) em 1. Enterococcus sp foi encontrado em 5 SSI (20,8\%), Escherichia coli e Klebsiella pneumoniae em 2 casos cada (8,3\%). Quanto às infecções do aparelho digestivo (AD) - 10 culturas positivas - Staphylococcus $s p$ foi isolado em $6(60 \%)$ pacientes (MRSA em 5 e MRSE-ENPC em 1 AD), Enterococcus $s p$ em 2 (20\%), Enterobacter cloacae e Acinetobacter baumanii em 1 caso cada. As infecções relacionadas à corrente sangüínea (BSI) - 12 culturas positivas - foram causadas principalmente por bacilos Gram-negativos (58,3\%). Os microorganismos isolados nas BSI foram: Klebsiella $s p$ e Staphylococcus sp em 3 casos (25\%) cada (MRSA em 2 e MRSE-ENPC em 1), Enterococcus faecalis, Escherichia coli e Acinetobacter baumanii em 2 casos cada (16,6\%), sendo um caso por Acinetobacter baumanii-PR. As infecções do trato urinário (UTI) - 14 culturas positivas - foram causadas por bacilos Gram-negativos em metade dos casos, cocos Grampositivos em 4 (28,5\%) e Candida sp em 3 (21,4\%). As bactérias mais freqüentemente isoladas foram: Enterococcus faecalis em 3 pacientes (21,4\%), Klebsiella pneumoniae e Pseudomonas aeruginosa-MR em 2 casos cada (14,3\%), Escherichia coli, MRSA, Citrobacter freudii e Enterobacter cloacae em 1 caso cada (7,1\%). Finalmente, as infecções do sistema cardiovascular (CVS) - 7 culturas positivas - relacionadas principalmente a cateteres intravenosos, foram devidas ao Staphylococcus $s p$ em 57,1\% dos casos (MSSA, MRSA, MRSE e MRSE-ENPC em 1 caso cada).

Dos pacientes que morreram, 18 (22,7\%) apresentaram infecção, sendo que cinco tinham pneumonia, quatro, infecção do aparelho digestivo, quatro, infecção relacionada à corrente sangüínea, três, infecção do sítio cirúrgico e dois, infecção urinária. A infecção relacionada à corrente sangüínea apresentou a maior taxa de letalidade (30,7\%), seguida pela infecção do aparelho digestivo $(29,4 \%)$ e pneumonia $(22,7 \%)$.

\section{DISCUSSÃO}

As complicações infecciosas ainda são bastante freqüentes em pacientes submetidos ao $\mathrm{TH}$, com média de 1,0 a 2,5 episódios de infecção por paciente, sendo ultrapassada somente pelas rejeições e disfunções do enxerto. As infecções bacterianas constituem a principal causa, sendo responsáveis por $35 \%$ a $70 \%$ das infecções em pacientes receptores, com média de 0,79 a 1,46 episódios por paciente transplantado. ${ }^{1-5,7-10}$ Dentre alguns fatores de risco apresentados pelos pacientes hepatopatas, além da própria insuficiência do órgão a ser transplantado, destacam-se as infecções latentes que podem ser reativadas quando o mesmo estiver sob imunossupressão, infecções ativas no momento do transplante, a complexidade do procedimento e o tempo cirúrgico despendido, bem como as condições clínicas freqüentemente precárias dos pacientes aguardando transplante. Assim, o risco de infecção após TH é resultado da combinação de fatores relacionados ao receptor, complicações cirúrgicas em geral, exposição ao ambiente e a imunossupressão pós-operatória ${ }^{6}$

Vários autores, em artigos de revisão, descreveram as principais características das complicações infecciosas em pacientes submetidos ao TH. ${ }^{6}{ }^{11}$ As infecções bacterianas intra-abdominais, de maneira geral, representam as complicações infecciosas mais comuns. As infecções de ferida cirúrgica, abscessos intraabdominais (intra ou extra-hepáticos), peritonites e colangites são as mais freqüentes e geralmente apresentam-se no primeiro mês pós-transplante. No nosso trabalho, as infecções bacterianas 
abdominais incluíram as IH de sítio cirúrgico (SSI) e do aparelho digestivo (AD), as quais, conjuntamente, apresentaram a maior incidência. Em relação aos abscessos e peritonites, alguns fatores de risco envolvidos são: trombose da artéria hepática, fístula biliar, laceração do enxerto e, mais raramente, perfuração intestinal. Os microorganismos isolados são, geralmente, bacilos gram-negativos, enterococos, anaeróbios e, raramente, Cândida sp. As colangites estão presentes em $4 \%$ a $16 \%$ dos pacientes transplantados e têm como principais fatores predisponentes, o tipo de anastomose biliar (colédoco-colédoco ou colédoco-jejuno, sendo mais freqüentes nesta última) e estenose da anastomose biliar.

A incidência de pneumonias após TH ocorre entre 13\% a 34\% dos casos, sendo responsável por $16 \%$ a $49 \%$ das infecções maiores nesses pacientes. As pneumonias bacterianas representam a maioria (40-67\%) das infecções pulmonares e, conforme o presente estudo, representam a segunda causa mais comum de infecções bacterianas após TH. Geralmente estão relacionadas à ventilação mecânica pós-operatória e, por conseguinte, ocorrem principalmente nos primeiros 30 dias após a cirurgia. As pneumonias nosocomiais são causadas predominantemente (60-67\%) por bacilos gram-negativos como Pseudomonas aeruginosa e enterobacteriaceas. O S. aureus, no entanto, vem se mostrando cada vez mais como um patógeno de importância nas pneumonias precoces pós-transplante. ${ }^{6,11}$

Bacteremias e infecções relacionadas à corrente sanguínea são encontradas em $19 \%$ a $25 \%$ dos receptores e representam $22 \%$ a $36 \%$ das complicações infecciosas maiores. ${ }^{1,2,9,10}$ Infecções intraabdominais constituem a fonte bacterêmica em $27 \%$ a $55 \%$ desses pacientes e as infecções relacionadas a cateteres intravenosos em $36 \%$ a $45 \%$. Os microorganismos mais freqüentemente isolados são cocos gram-positivos (Staphylococcus aureus, Enterococcus sp e Staphylococcus coagulase-negativos) presentes em $40 \%$ a $59 \%$ das bacteremias, seguidos por germes gram-negativos - conseqüentes às infecções intra-abdominais (33-48\%) e pulmonares (14-17\%). Tendo em vista a elevada mortalidade desses pacientes (25-35\%), uma terapêutica antibiótica empírica ampla deve ser prontamente iniciada nesses casos, bem como a realização de uma investigação da provável complicação clínica ou cirúrgica subjacente. ${ }^{6,11,12} \mathrm{Em}$ nosso levantamento, as BSI apresentaram-se com incidência menor à relatada na literatura. Isso provavelmente deveu-se à classificação topográfica específica da $\mathrm{IH}$, sempre que o foco responsável pela bacteremia era identificado, excluindo-o da categoria BSI.

Em relação às infecções fúngicas, elas ocorrem com maior incidência nos pacientes submetidos ao $\mathrm{TH}$, em relação aos outros transplantes de órgãos sólidos. ${ }^{13}$ Atingem $9 \%$ a $42 \%$ dos receptores, sendo a Candida $s p$ responsável por $77 \%$ a $83 \%$ e o Aspergillus $s p$ por $15 \%$ a $20 \%$ de todas as infecções fúngicas. ${ }^{2,13-17}$ As candidíases geralmente apresentam-se como infecções superficiais de pele ou cavidade oral, esofagite, infecção de ferida cirúrgica, abscesso intraabdominal, peritonite, fungemias relacionadas a cateter ou mesmo sem foco definido; e a grande maioria ocorre nos dois primeiros meses pós-transplante. Alguns fatores de risco reconhecidos incluem: utilização excessiva de corticóides e antibióticos no pré-operatório, infecção por citomegalovírus, colonização por fungos no momento do transplante, tempo cirúrgico prolongado, grandes perdas sanguíneas, insuficiência renal e necessidade de reoperações ou retransplante. Comparadas às infecções virais e bacterianas nesses pacientes, as infecções fúngicas apresentam uma mortalidade muito maior $(50-75 \%)$, chegando próximo de $100 \%$ nos casos de aspergilose. ${ }^{6,11}$

A despeito de, muitas vezes, as taxas de IH em hospitais universitários estarem relacionadas à aprendizagem do novo corpo discente (acadêmicos de medicina e enfermagem, médicos residentes, entre outros), sendo maior no início de cada ano, isso não foi observado em nosso serviço. ${ }^{18}$ Os meses de maior incidência, entretanto, exceto julho, coincidiram com o período mais frio do ano. ${ }^{19-20}$ Tendo em vista que os hospitais e residências em Curitiba não são aclimatizados, a temperatura mais baixa na cidade predispõe às infecções pulmonares.

Não obstante a elevada incidência das infecções bacterianas em pacientes transplantados hepáticos, graças à identificação de fatores de risco pré-operatórios, utilização de novos esquemas imunossupressores e instituição de medidas preventivas cada vez mais eficazes, a mortalidade pós-TH relacionada às infecções tem diminuído progressivamente. ${ }^{6}$ Antes da década de 80 , mais da metade desses pacientes evoluía a óbito, incidência essa reduzida para $25-35 \%$ na década de 80 e para menos de $10 \%$ em muitos centros, após $1990 .{ }^{6}$ Esses números certamente refletem, embora não de maneira isolada, a melhora progressiva da taxa de sobrevida observada no primeiro ano após o transplante, a qual era menor que $40 \%$ antes de 1980 , elevando-se para aproximadamente $70 \%$ na década de 80 e para mais de $80 \%$ a partir de $1990 .^{6}$ A taxa de mortalidade em nosso estudo, envolvendo também pacientes pré-TH com IH, foi de $22,7 \%$. Essa incidência, de certa forma elevada, provavelmente deve-se à grande freqüência das infecções hospitalares por microorganismos resistentes $(43 \%$ das $\mathrm{IH}$ ). Corroborando a isso, inclui-se no grupo pacientes prétransplante com variados graus de insuficiência hepática e, por vezes, insuficiência de outros órgãos, com condição clínica bastante comprometida.

Apesar da maior taxa de infecção bacteriana em pacientes de serviço de transplante hepático ser relacionada ao sítio cirúrgico, a maior taxa de letalidade é devida às infecções primárias da corrente sangüínea, seguida pelas infecções do aparelho digestivo. As bactérias isoladas mais freqüentes são Staphylococcus $s p$ e Enterococcus faecalis, sendo que um número expressivo destas bactérias são multirresistentes. Isso reforça a importância da identificação e controle dos fatores de risco envolvidos, bem como medidas preventivas para as infecções hospitalares, uso criterioso dos antimicrobianos, supervisão ativa dos serviços de controle de infecção hospitalar e isolamento dos pacientes contaminados ou infectados, a fim de se reduzirem as infecções por microorganismos resistentes e, por conseguinte, a taxa de mortalidade.

\section{CONCLUSÃO}

O local mais comum de infecção bacteriana em pacientes de serviço de transplante hepático é o sítio cirúrgico e a causa de maior letalidade é a infecção da corrente sangüínea. 


\section{ABSTRACT}

Objective: The incidence of infection after liver transplantation remains high, being even higher if compared to other solid-organs transplantations. The aim of this study is to report the incidence, topographic distribution and the most frequently isolated microorganisms in nosocomial infections in our liver transplantation unit. Methods: medical charts of 1,963 patients admitted for liver transplantation or for pre or post transplantation complications were evaluated prospectively using the diagnostic criteria for nosocomial infections employed by the Center for Disease Control and Prevention (CDC) of the United States of America. During the period of time of the study, 175 liver transplantations were performed (148 with cadaveric donors and 27 with living donors). Results: nosocomial infections were diagnosed in 79 patients, representing $5.5 \%$ of the total patient admissions. The greatest incidence of infection was at the surgical site $(27.7 \%$ - 30 cases), followed by pneumonia (20.3\%), gastrointestinal infections (15.7\%) and blood stream related infections (12\%). The most common bacteria isolated in 79 positive cultures were: Staphylococcus sp (40.5\%), Enterococcus faecalis (13.9\%), Klebsiella pneumoneae and Escherichia coli ( $76 \%$ each). Among the infections caused by multiresistant bacteria ( $43 \%$ of the total), methicilin-resistant Staphylococcus aureus (MRSA) was the most prevalent (22 infections). The global mortality related to nosocomial infections was $22.7 \%$ (18 patients) and the most lethal infection was related to the bloodstream (30.7\%). Conclusion: the most common site of bacterial infection in liver transplantation unit patients is surgical site infection and the most lethal infection is related to the bloodstream. The most frequent bacteria isolated were Staphylococcus $s p$ and Enterococcus faecalis, being a great number of them multiresistent.

Keywords: Nnosocomial infection, Bacteria, Pneumonia, Liver transplantation, Hepatic disease

\section{REFERÊNCIAS}

1. Colonna JO, Winston DJ, Brill JE, Goldstein LI, Hoff MP, Hiatt JR, et al. Infectious complications in liver transplantation. Arch Surg 1988;123:360-4.

2. Kusne S, Dummer JS, Singh N, Iwatsuki S, Makowka L, Esquivel C, et al. Infections after liver transplantation. An analysis of 101 consecutive cases. Medicine (Baltimore) 1988;67:132-43

3. Paya CV, Hermans PE, Washington JÁ 2nd, Smith TF, Anhalt JP, Wiesner RH, et al. Incidence, distribution, and outcome of episodes of infection in 100 orthotopic liver transplantation. Mayo Clin Proc 1989;64:555-64.

4. Saint-Vil D, Lux FI, Lebel P, Brandt ML, Paradis K, Weber A, et al. Infectious complications of pediatric liver transplantation. J Pediatr Surg 1991;26:908-13.

5. George DL, Arnow PM, Fox A, Thistlethwaite JR, Emond JC, Broelsch CE, et al. Patterns of infection after pediatric liver transplantation. Am J Dis Child 1992;146:924-9.

6. Winston DJ, Emmanouilides C, Busuttil RW. Infections in liver transplant recipients Clin Infect Dis 1995;21:1007-89.

7. George DL, Arnow PM, Fox AS, Thistlethwaite JR, Emond JC, Whitington PF, et al. Bacterial infection as a complication of liver transplantation: epidemiology and risk factors. Rev Infect Dis 1991;13:387-96.

8. Lumbreras C, Lizasoain M, Moreno E, Aguado JM, Gomez R, Garcia I, et al. Major bacterial infection following liver transplantation: a prospective study Hepatogastroenterology 1992;39:362-5.

9. Singh N, Gayorvski T, Warner MM, Marino IR. Increased infections in liver transplant recipients with recurrent hepatitis $\mathrm{C}$ virus hepatitis. Transplantation 1996;61:396-401.

10. Wade JJ, Rolando N, Hayllar K, Philpott-Howard J, Casewell MW, et al. Bacterial and fungal infections after liver transplantation: an analysis of 284 patients. Hepatology 1995;21:1328-36.

11. Singh N. Infectious diseases in liver transplant recipient. Sem Gastrintest Dis 1998;9:136-46

12. Wagener MM, Yu VL. Bacteremia in transplant recipients: a prospective study of demographics, etiologic agents, risk factors, and outcomes. Am J Infect Control 1992;20:239-47.

13. Paya CV. Fungal infections in solid-organ transplantation. Clin Infect Dis 1993;16:677-88.

14. Schroter GP, Hoelscher M, Putnam CW, Porter KA, Starzl TE. Fungus infections after liver transplantation. Ann Surg 1977;186:115-22.

15. Wajszczuk CP, Dummer JS, Ho M, Van Thiel DH, Starzl TE, Iwatsuki S, Shaw B Jr. Fungal infections in liver transplant recipients. Transplantation 1985;40:347-53.

16. Castaldo P, Stratta RJ, Wood RP, Markin RS, Patil KD, Shaefer MS. Clinical spectrum of fungal infections after orthotopic liver transplantation. Arch Surg 1991;126:149-56.

17. Collins LA, Samore MH, Roberts MS, Luzzati R, Jenkins RL, Lewis WD. Risk factors for invasive fungal infections complicating orthotopic liver transplantation. J Infect Dis 1994;170:644-52.

18. Ferraz EM. Inquérito nacional sobre infecções pós-operatórias. Rev Col Bras Cir 1981;8:253-62.

19. Raad I, Abbas J, Whimhey E. Infection control of nosocomial respiratory viral disease in the immunocompromised host. Am J Med 1997;102:48-54.

20. Palmer SM, Henshaw NG, Howell DN, Miller SE, Davis RD et al. Community respiratory viral infection in adult lung transplant recipients. Chest 1998;113:944-50. 579-81

21. Adamec M. Removal of the kidney from a related transplant donor using subcostal lumbotomy. Rozhl Chir 1995 Sep; 74(6): 302-4 